\title{
Semi-automatic Categorization of Videos on VideoLectures.net
}

\author{
Miha Grcar $^{1}$, Dunja Mladenic ${ }^{1}$, and Peter Kese $^{2}$ \\ ${ }^{1}$ Jozef Stefan Institute, Dept. of Knowledge Discovery, Jamova cesta 39, \\ 1000 Ljubljana, Slovenia \\ \{miha.grcar, dunja.mladenic\}@ijs.si \\ ${ }^{2}$ Viidea Ltd., Glavni trg 25, \\ 4000 Kranj, Slovenia \\ peter.kese@viidea.com
}

\begin{abstract}
Automatic or semi-automatic categorization of items (e.g. documents) into a taxonomy is an important and challenging machine-learning task. In this paper, we present a module for semi-automatic categorization of video-recorded lectures. Properly categorized lectures provide the user with a better browsing experience which makes her more efficient in accessing the desired content. Our categorizer combines information found in texts associated with lectures and information extracted from various links between lectures in a unified machinelearning framework. By taking not only texts but also the links into account, the classification accuracy is increased by $12-20 \%$.
\end{abstract}

Keywords: categorization, classification, machine learning, multi-modal data mining, multimedia, video, VideoLectures.net.

\section{Introduction and Motivation}

In this paper, we present a module for semi-automatic categorization of videorecorded lectures. The presented categorizer is part of the software powering one of the world's largest educational Web portals, the VideoLectures video-hosting portal ${ }^{1}$.

VideoLectures is growing fast. There are roughly 200 videos added each month. In order to provide the audience with efficient browsing facilities, each video needs to be categorized into one or more categories in the taxonomy. The taxonomy now has roughly 150 categories and is still growing. The categorization of lectures is a timeconsuming task and is far from trivial.

Our categorizer combines information found in texts associated with lectures (e.g. title, description, slide texts) and information extracted from various links between lectures (e.g. lectures recorded at the same event, lectures sharing an author, lectures interlinked according to the click-stream data) in a unified machine-learning framework. The VideoLectures team provided us with their dataset of English lectures; roughly one third of them were already manually categorized and served as

${ }^{1}<$ http://videolectures.net $>$ 
training data. Each lecture is described with a title; more than two thirds of the lectures also have a short description and/or come with slide titles. In addition to this unstructured textual data, each lecture can be seen as a vertex in several undirected weighted graphs representing the structural part of the data. These graphs are the following:

- Same-event graph. Two vertices are interconnected if the two corresponding lectures were recorded at the same event (e.g. a specific conference or workshop).

- Same-author graph. Two vertices are interconnected if the two corresponding lectures were prepared and/or given by the same author or authors. The weight of a link is the number of authors the two lectures have in common.

- Also-watched graph. Two vertices are interconnected if the two corresponding lectures were viewed together in the same browsing session. The weight of a link is the number of times the two lectures were viewed together.

The categorizer has already been integrated into the VideoLectures Web site. When the author or the editor decides to categorize a lecture, she is provided with a set of suggested categories (called "quick links" in the categorization panel). These suggestions were pre computed by the categorizer. The author/editor simply selects the appropriate categories in contrast to browsing the taxonomy to find them. The categorization user interface is shown in Fig. 1.

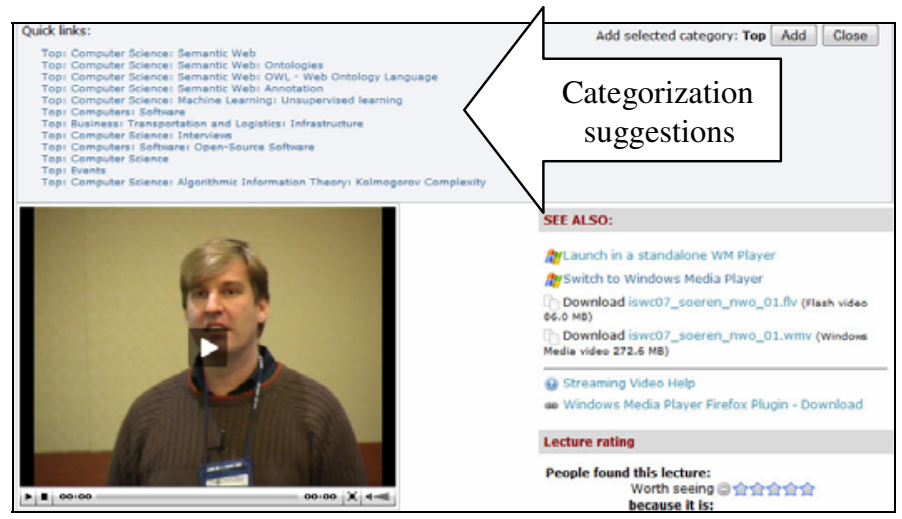

Fig. 1. Categorization user interface on VideoLectures

\section{Approach and Experimental Results}

Classification of documents into a taxonomy has already been addressed by several researchers, either on a flattened taxonomy [2] or by taking the structure into account [3]. We decided to flatten the taxonomy and employ Centroid Classifier as suggested in [1].

To evaluate the approach, we first represented each lecture by the associated text as TF-IDF vector as usual in text mining and performed 5-fold cross validation on the manually categorized lectures. We measured classification accuracies on top 1, 3, 5, 
and 10 predicted categories. We tested several classifiers: the Centroid, SVM, and $k$-Nearest Neighbors $(k$-NN) classifier. From the results, we learned that the three algorithms perform comparably well in terms of accuracy, with the Centroid Classifier standing out with respect to the accuracy on top 10 items (achieving $83.48 \%$ accuracy on average) and SVM achieving a slightly better accuracy on the topmost item (achieving 53.89\% accuracy on average). Regarding the efficiency, it is important to note that SVM is slow in the training phase while $k$-NN is slow in the prediction phase. These two facts give even more appeal to the Centroid Classifier which was our choice for further experimentation and also final implementation.

To incorporate structural data in an attempt to boost accuracy, we decided to compute a kernel for each different "instance space" of the data, different spaces being the TF-IDF representation of the textual data on one hand and the three undirected weighted graphs (i.e. same-event, same-author, and also-watched graph) on the other.

We can look at a set of normalized TF-IDF vectors $\mathbf{f}_{i},\left\|\mathbf{f}_{i}\right\|_{2}=1, f_{i, j} \geq 0$, as if they are rows in matrix $\mathbf{F}_{\mathrm{TF}-\mathrm{IDF}}=\left[f_{i, j}\right]$. If so, the corresponding kernel $\mathbf{K}_{\mathrm{TF}-\mathrm{IDF}}$ is obtained by multiplying $\mathbf{F}_{\text {TF-IDF }}$ with the transposed form of itself. When dealing with data in the form of an undirected weighted graph, on the other hand, we compute a diffusion kernel [4]. Let us consider attaching a random variable $Z_{i}$ to each vertex $i$. Now let each variable send some of its value (fraction $\alpha$ ) to each of the immediate neighbors at discrete time steps. It turns out that the covariance matrix of such random field is a kernel reflecting similarities between vertices: the more two vertices $i$ and $j$ are interconnected in a graph, the more of $Z_{i}$ is transitioned to $Z_{j}$ and vice versa. Covariance between $i$ and $j$ is consequently increased.

When we compute all the kernels (i.e. the TF-IDF kernel and the diffusion kernels), we "join" them together into a single kernel by computing a convex combination of kernels $\mathbf{K}_{\Sigma}=\alpha_{1} \mathbf{K}_{\mathrm{TF}-\mathrm{IDF}}+\alpha_{2} \mathbf{K}_{\mathrm{G}_{1}}+\alpha_{3} \mathbf{K}_{\mathrm{G}_{2}}+\alpha_{4} \mathbf{K}_{\mathrm{G}_{3}} \cdot \mathbf{K}_{\Sigma}$ is again a kernel.

The task is now to set the weights $\alpha_{i}$ so that a high accuracy is achieved. For this purpose, we split the dataset into a training and test set for each fold as we did when

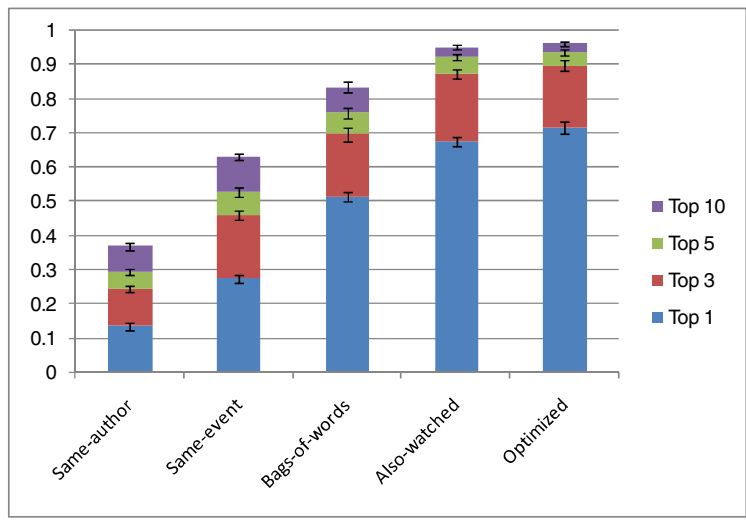

Fig. 2. Classification accuracies for each type of information in isolation as well as for the optimized combined kernels 
establishing the baseline. However, we then run an optimization algorithm called Differential Evolution [5] to optimize the convex combination weights. In the optimization loop, we evaluate the intermediate weights by performing another (inner) 5-fold cross validation.

The accuracy of the optimized convex combination as well as the accuracies of the baseline and each of the graphs in isolation are shown in Fig. 2. From the chart, we can conclude the following. The same-author and same-event graphs contain the least relevant information for our categorization task. The same-event graph performs a bit better than same-author but is still below the baseline. By far the most relevant information is contained in the also-watched graph. This graph, in isolation, outperforms the other graphs as well as the text-based baseline. The optimized feature kernel indeed yields the best accuracies. However, the performance of the optimized data representation is close to that of the also-watched graph-based representation.

\section{Conclusions}

We can conclude that we were successful in our task as the categorizer is highly accurate- the proposed approach using text and graph features achieves accuracies that stretch 12-20\% above the baseline obtained using text features only-and highly robust in terms of missing data. The latter means that a lecture might be missing textual annotations (such as the description and slide titles) but is still categorized correctly. Furthermore, the categorizer has been successfully integrated into the VideoLectures Web site. Categorization suggestions (termed "quick links") are provided to the author in the categorization panel.

The current implementation of the categorizer suffers from high memory consumption as each diffusion kernel is a $n \times n$ dense matrix, where $n$ is the number of lectures. Also, computing diffusion kernels is a time-consuming operation. We are currently working on reducing these time-space requirements to make the categorizer more scalable.

\section{References}

1. Grobelnik, M., Mladenic, D.: Simple Classification into Large Topic Ontology of Web Documents. Journal of Computing and Information Technology 13, 279-285 (2005)

2. Koller, D., Sahami, M.: Hierarchically Classifying Documents Using Very Few Words. In: Proceedings of the 14th International Conference on Machine Learning ICML 1997, pp. 170-178. Morgan Kaufmann, San Francisco (1997)

3. Mladenic, D.: Turning Yahoo into an Automatic Webpage Classifier. In: Proceedings 13th European Conference on Artificial Intelligence ECAI 1998, pp. 473-474. John Wiley \& Sons, Chichester (1998)

4. Kondor, R.I., Lafferty, J.: Diffusion Kernels on Graphs and Other Discrete Structures. In: Proceedings of the ICML 2002, pp. 315-322 (2002)

5. Storn, R., Price, K.: Differential Evolution - A Simple and Efficient Heuristic for Global Optimization over Continuous Spaces. Journal of Global Optimization 11, 341-359 (1997) 